\title{
ON ARCHIMEDEAN ORDERED VECTOR SPACES AND A CHARACTERIZATION OF SIMPLICES
}

\author{
GERHARD GIERZ AND BORIS SHEKHTMAN
}

(Communicated by William J. Davis)

\begin{abstract}
We show that a convex subset $K$ of a linear space is a simplex if and only if it is line compact and every nonempty intersection of two translates of $K$ is a homothet of $K$. This answers a problem posed by Rosenthal. The proof uses a reformulation of this problem in terms of Archimedean ordered spaces
\end{abstract}

\section{INTRODUCTION}

Let $K$ be a convex subset of a linear space $E$. If $K \times\{1\}$ is the base for a lattice cone in $X \times \Re$, then $K$ is called a simplex (see [7] for the definitions). A remarkable result of Kendall [4] shows that $K$ is a simplex if and only if $K$ is line-compact ${ }^{1}$ and the nonempty intersection of two homothets ${ }^{2}$ of $K$ is a homothet of $K$. Moreover, every simplex has the property that it is line compact and every nonempty intersection of two translates of $K$ is a homothet of $K$. It is an open problem whether this last condition fully characterizes simplices, but a result of Rosenthal [7] shows that this is the case at least for $\sigma$ convex subsets of topological vector spaces. Using Archimedean ordered spaces and their known relation to simplices, Rosenthal [7] reformulated this open problem as follows.

Let $E$ be an ordered vector space. Given a positive function $\mu: E \rightarrow \Re$, we say that $E$ has a $\mu$-lattice structure if $\mu\left(u_{1}\right)=\mu\left(u_{2}\right)$ implies that $u_{1} \vee u_{2}$ exists in $E$.

Note that in an ordered vector space the existence of $-u_{1} \vee H-u_{2}$ implies that $u_{1} \wedge u_{2}$ exists and is equal to $-\left(-u_{1} \vee-u_{2}\right)$. Hence in a $\mu$-lattice the infimum $u_{1} \wedge u_{2}$ exists whenever $\mu\left(u_{1}\right)=\mu\left(u_{2}\right)$.

Recall that a positive functional $\mu$ is called strictly positive if $u \geq 0$ and $\mu(u)=0$ implies that $u=0$.

Rosenthal has shown that an affirmative answer to the following question leads to a positive solution of the open problem concerning simplices as stated above.

Received by the editors December 4, 1990 and, in revised form, February 21, 1991.

1991 Mathematics Subject Classification. Primary 46A55, 46A40.

Key words and phrases. Simplices, Archimedean ordered spaces, sublattices of $C(K)$.

${ }^{1} K$ is line-compact if the intersection of every line with $K$ is compact.

${ }^{2} \mathrm{~A}$ homothet of $K$ is a set of the form $a+r \cdot K$, where $r \geq 0$ is a positive constant. 
Problem. Let $E$ be an Archimedean ordered space. If $E$ has a $\mu$-lattice structure for a strictly positive linear functional $\mu$ on $E$, is it necessarily a vector lattice?

In this note we give an affirmative answer to this problem (Theorem 3.5).

For definitions and results concerning vector lattices and Banach lattices, see $[5,3]$.

\section{2. $\mu$-Sublattices of $C(K)$}

In the following, let $E \subseteq C([0,1])$ be a subspace such that (i) $1, x \in E$ and (ii) there is a positive measure $\mu$ on $[0,1]$ such that $0,1 \in \operatorname{supp}(\mu)$ and such that for all $f, g \in E$ with $\mu(f)=\mu(g)$ we have $\sup (f, g) \in E$. We would like to show that $E$ contains all piecewise linear functions.

We need some notation. For a point $a \in[0,1]$, define maps $\lambda_{a}, \rho_{a} \in$ $C([0,1])$ by

$$
\lambda_{a}(x)= \begin{cases}a-x & \text { if } x \leq a \\ 0 & \text { else }\end{cases}
$$

and

$$
\rho_{a}(x)= \begin{cases}x-a & \text { if } x \geq a, \\ 0 & \text { else }\end{cases}
$$

Since $\lambda_{a}(x)-\rho_{a}(x)=a-x=a \cdot 1-x$, we have $\lambda_{a} \in E$ if and only if $\rho_{a} \in E$. Also, since $\lambda_{a}(x)=\sup \{(a \cdot 1-x), 0)$, condition (ii) implies that there is a number $a_{0}$ with $0<a_{0}<1$ such that $\lambda_{a_{0}}, \rho_{a_{0}} \in e$.

2.1. Proposition. Let $E \subseteq C([0,1])$ be a subspace such that

(i) $1, x \in E$;

(ii) there is a positive measure $\mu$ on $[0,1]$ such that $0,1 \in \operatorname{supp}(\mu)$ and such that $E$ is a $\mu$-lattice.

Then $E$ contains all piecewise linear functions.

Proof. Every piecewise linear function is a linear combination of functions of the forms $\lambda_{b}$ and $\rho_{b}, 0<b<1$. Hence, we have to show that $\lambda_{b}, \rho_{b} \in E$ for all $b$ with $0<b<1$. Let $a_{0}$ be a number with $0<a_{0}<1$ such that $\lambda_{a_{0}}$, $\rho_{a_{0}} \in E$, and let $0<b<1$ be given. We shall assume that $a_{0}<b$; the case where $b<a_{0}$ is treated similarly.

Consider the function $f$ given by $f(x)=x-b$ and let $r=\int f(x) d \mu$. Then, since $\mu$ is positive and $0 \in \operatorname{supp}(\mu)$, it follows that $\int \lambda_{a_{0}}(x) d \mu>0$, and we can find a number $s$ such that

$$
r=s \int \lambda_{a_{0}}(x) d \mu .
$$

From our assumptions on $E$ it follows that

$$
g_{1}=\sup \left(f, s \cdot \lambda_{a_{0}}\right)-s \cdot \lambda_{a_{0}} \in E .
$$

We will consider two cases:

(i) $-b / a_{0} \leq s$. In this case, $\rho_{b}=g_{1} \in E$.

(ii) $s<-b / a_{0}$. In this case, $g_{1}=r_{1} \cdot \lambda_{a_{1}}+\rho_{b}$ where

$$
r_{1}=-(1+s) \quad \text { and } \quad a_{1}=\frac{b+s a_{0}}{1+s} \leq a_{0} .
$$


Let

$$
R=\int \rho_{b} d \mu, \quad S=\int \lambda_{a_{0}} d \mu .
$$

Since $\rho_{b} \leq g_{1}$, we have $\int g_{1} d \mu \geq R$. Again, we can find a number $s_{1}$ so that

$$
s_{1} \int \lambda_{a_{0}} d \mu=\int g_{1} d \mu
$$

and it follows that $s_{1} \geq R / S$. It follows that $a_{0} \cdot R / S \leq s_{1} \cdot a_{0}=s_{1} \cdot \lambda_{a_{0}}(0)$. Define

$$
g_{2}=\sup \left(g_{1}, s_{1} \cdot \lambda_{a_{0}}\right)-s_{1} \cdot \lambda_{a_{0}} .
$$

Then $g_{2} \in E$, and either $g_{2}=\rho_{b} \in E$ (in the case where $s_{1} \cdot a_{0} \geq r_{1} \cdot a_{1}$ ) or $g_{2}=r_{2} \cdot \lambda_{a_{2}}+\rho_{b}$ where $a_{2} \leq a_{1} \leq a_{0}$ and

$$
r_{2} \cdot a_{2}=r_{1} \cdot a_{1}-s_{1} \cdot a_{0} \leq r_{1} \cdot a_{1}-(R / S) \cdot a_{0} \text {. }
$$

We continue in this way with $r_{2}$ and $a_{2}$ in the place of $r_{1}$ and $a_{2}$ until we finally find an index $n$ such that $s_{n} \cdot a_{0} \geq r_{n} \cdot a_{n}$, and therefore $g_{n+1}=\rho_{b} \in E$. Note that this procedure has to terminate after finitely many steps since

$$
r_{n} \cdot a_{n} \leq r_{n-1} \cdot a_{n-1}-(R / S) \cdot a_{0} \quad \text { and } \quad s_{n} \geq R / S \text {. }
$$

In the following result, we generalize the domain of the functions slightly. Before we do this, let us make some remarks concerning positive measures. Let $K$ and $K^{\prime}$ be compact Hausdorff spaces, and let $\phi: K \rightarrow K^{\prime}$ be a continuous map. If $\nu$ is a measure on $K$, then define a measure $\nu^{\prime}$ on $K^{\prime}$ by

$$
\int d d \nu^{\prime}=\int(f \circ \phi) d \nu
$$

Then $\nu^{\prime}=T_{\phi}^{*}(\nu)$, where $T_{\phi}^{*}$ is the adjoint to the operator

$$
\begin{aligned}
T_{\phi}: C\left(K^{\prime}\right) & \rightarrow C(K), \\
f & \mapsto f \circ \phi .
\end{aligned}
$$

The supports of $\nu$ and $\nu^{\prime}$ are related by the equation

$$
\operatorname{supp}\left(\nu^{\prime}\right)=\phi(\operatorname{supp}(\nu)) \text {. }
$$

2.2. Proposition. Let $E \subseteq C([a, b])$ be a subspace such that

(i) $1, x \in E$;

(ii) there is a positive measure $\mu$ on $[0,1]$ such that $a, b \in \operatorname{supp}(\mu)$ and such that $E$ is a $\mu$-lattice.

Then $E$ contains all piecewise linear functions.

Proof. Define a map

$$
\phi:[0,1] \rightarrow[a, b], \quad x \mapsto a+(b-a) \cdot x .
$$

Then the map

$$
T_{\phi}: C([a, b]) \rightarrow C([0,1]), \quad f \mapsto f \circ \phi
$$

is a (norm-preserving) linear bijection that respects the lattice structure of $C([a, b])$ and that also sends the space of all piecewise linear functions on $C([a, b])$ onto the space of all piecewise linear functions on $C([0,1])$. Moreover, $\mu$ is a positive measure on $C([0,1])$ if and only if it is of the form 
$\mu=T_{\phi}^{*}(\nu)$, where $\nu$ is a positive measure on $[a, b]$. Furthermore, since $\operatorname{supp}(\mu)=\phi(\operatorname{supp}(\nu))$, we have $0,1 \in \operatorname{supp}(\mu)$ if and only if $a, b \in \operatorname{supp}(\nu)$. Hence Proposition 2.2 follows from 2.1.

2.3. Theorem. Let $K$ be a compact Hausdorff space, let $E \subseteq C(K)$ be a linear subspace containing a strictly positive function $e$, and let $\mu$ be a strictly positive measure on $K$ such that $E$ is a $\mu$-lattice. Then $E$ is a sublattice of $C(K)$.

Proof. First, consider the map

$$
T_{e^{-1}}: C(K) \rightarrow C(K), \quad f \mapsto e^{-1} \cdot f .
$$

This map is linear, bijective, and preserves the lattice structure. Hence, we may replace $E$ by $T_{e^{-1}}(E), e$ by 1 , and $\mu$ by $e \cdot \mu$ in the statement of the theorem. It follows that we may assume w.l.o.g. that $1 \in E$.

Now let $\phi \in E$ be arbitrary. We have to show that $|\phi| \in E$. Let

$$
a=\min _{0 \leq \max \leq 1} \phi(x), \quad b=\max _{0 \leq x \leq 1} \phi(x) .
$$

Consider the linear operator

$$
\begin{aligned}
T_{\phi}: C([a, b]) & \rightarrow C(K) \\
f & \mapsto f \circ \phi .
\end{aligned}
$$

Again, this operator is linear and a lattice homomorphism (in the sense that $T_{\phi}(|f|)=\left|T_{\phi}(f)\right|$ for all continuous $\left.f \in C([a, b])\right)$. Let $F=T_{\phi}^{-1}(E)$ and let $\mu^{\prime}=T_{\phi}^{*}(\mu)$. Then $F$ is a linear subspace of $C([a, b])$ with $1 \in F$ (since $\left.T_{\phi}(1)=1 \circ \phi=1\right)$, and we have $x \in F$; indeed, $x \in F$ means that $i \in F$, where $i(x)=x$. But $i \in F$ is equivalent to $T_{\phi}(i)=i \circ \phi=\phi \in E$, and the last statement is true by our assumptions. Moreover, $\mu^{\prime}$ is positive; if $f \in C[a, b]$ is positive, then $f \circ \phi$ is positive, and we obtain $\mu^{\prime}(f)=\mu(f \circ \phi) \geq 0$. Since $\mu$ is strictly positive, $\operatorname{supp}(\mu)=K$, hence $\operatorname{supp}\left(\mu^{\prime}\right)=\phi(K)$. It follows that $a$, $b \in \operatorname{supp}\left(\mu^{\prime}\right)$. Lastly, assume that $f, g \in F$ and that $\mu^{\prime}(f)=\mu^{\prime}(g)$. Then $\mu\left(T_{\phi}(f)\right)=\mu\left(T_{\phi}(g)\right)$ and $T_{\phi}(f), T_{\phi}(g) \in E$. Hence, by our assumptions on $E$, we conclude that $T_{\phi}(\sup (f, g))=\sup \left(T_{\phi}(f), T_{\phi}(g)\right) \in E$, and therefore $\sup (f, g) \in F$. It follows from Proposition 2.2 that $F$ contains all piecewise linear functions. In particular, $|i| \in F$ (where $|i|(x)=|x|$ for all $a \leq x \leq b)$. By the definition of $F$, we conclude that $|\phi|=\left|T_{\phi}(i)\right|=T_{\phi}(|i|) \in E$.

2.4. Proposition. Let $E \subseteq C(K)$ be a subspace such that $1 \in E$. Assume that $E$ separates the points of $K$. Further, let $\phi: E \rightarrow \Re$ be a strictly positive functional on $E$ such that $E$ is a $\phi$-lattice. Then $\phi$ can be extended to a strictly positive linear functional $\phi^{\prime}: C(K) \rightarrow \Re$.

Proof. Firstly, we may assume that $\phi(1)=1$. By the vector lattice version of the Hahn-Banach Theorem (see Proposition II.4.4 of [3] with $U=\{f \in$ $C(K):-1 \leq f \leq 1\}$ ), we can extend $\phi$ to a positive functional $\phi^{\prime}: C(K) \rightarrow \Re$, and it suffices to show that each such extension is strictly positive. Actually, there is only one such extension, since it will follow later that $E$ is dense in $C(K)$, and the density of $E$ in $C(K)$ implies that this extension has to be strictly positive, but we cannot use the density of $E$ at this point.

Since every positive functional is bounded and hence given by a measure on $K$, we find that $\phi^{\prime}(f)=\int f d \mu$ for some positive measure $\mu$ on $K$. In order 
to show that $\phi^{\prime}$ is strictly positive, we have to show that $\operatorname{supp}(\mu)=K$. Assume not, and let $x_{0} \in K \backslash \operatorname{supp}(K)$. We will construct a positive element $f_{0} \in E$ such that $f_{0}\left(x_{0}\right)>0$ and $f_{0}(y)=0$ for all $y \in \operatorname{supp}(\mu)$. This will lead to the contradiction $0<\phi\left(f_{0}\right)=\phi^{\prime}(f)=\int f_{0} d \mu=\int_{\operatorname{supp}(\mu)} f_{0} d \mu=0$.

As a first step, we show that for every $x \in K$ there is an open neighborhood $U$ of $x$ and a positive element $f \in E$ such that $0 \neq f$ and $f(y)=0$ for all $y \in U$. In order to construct $f$, pick any nonconstant function $g \in E$ and consider the element $g-\phi(g) \cdot 1$. There are two cases to consider.

1. $g(x) \neq \phi(g)$. If $g(x)<\phi(g)$, then since $\phi(g-\phi(g) \cdot 1)=0$, the element $f=(g-\phi(g) \cdot 1)_{+}=(g-\phi(g) \cdot 1) \vee 0$ belongs to $E$ and is 0 on a neighborhood of $U$. Moreover, since $g$ is not constant, $g-\phi(g) \cdot 1 \neq 0$. Since $g-\phi(g) \cdot 1<0$ would imply that $\phi(g-\phi(g) \cdot 1)<0$, we conclude that $g-\phi(g) \cdot 1$ is not negative, hence there is at least one point $x_{1}$ for which $f\left(x_{1}\right)=(g-\phi(g) \cdot 1)\left(x_{1}\right)>0$. We conclude that $0<f$. If $g(x)>\phi(g)$, then we replace $g$ by $-g$.

2. $g(x)=\phi(g)$. In this case, the same construction as in case (1) delivers a function $f^{\prime}>0$ such that $f^{\prime}(x)=0$. We now replace $g$ by $f^{\prime}$, and for this new $g$ we have $g(x)=0<\phi(g)$. Hence case (1) applies to the new function $g$, and we also find a function $f>0$ such that $f(y)=0$ for all $y$ in a neighborhood of $x$.

In the next step, we show that for every $x \in \operatorname{supp} \mu$ there is a function $f_{x} \in E$ such that $0 \leq f_{x}, f_{x}\left(x_{0}\right)>0$ and such that $f_{x}$ vanishes on a neighborhood $U_{x}$ of $x$. In order to construct $f_{x}$, pick any function $g$ such that $g\left(x_{0}\right) \neq g(x)$. Such a function exists since $E$ separates the points of $K$. After subtracting a multiple of the constant function 1 and multiplying by -1 , if necessary, we may assume that $g\left(x_{0}\right)>0>g(x)$. Let $r=\phi\left(g_{0}\right)$. If $r \geq 0$, then pick any positive function $0 \neq h \in E$ with $h\left(x_{0}\right)=0$ and $\phi(h)=1$. If $r \leq 0$, then pick a negative function $0 \neq h \in E$ that vanishes on a neighborhood of $x$ and satisfies $\phi(h)=-1$. In both cases, the function $h$ exists by the previous step. Now let $g^{\prime}=g-|r| \cdot h$. Then $\phi\left(g^{\prime}\right)=0, g^{\prime}\left(x_{0}\right)>0$, and $g^{\prime}$ is negative on a neighborhood of $x$. Hence, by our assumptions on $E, f_{x}=g^{\prime} \vee 0 \in E$, $f_{x}\left(x_{0}\right)>0$, and $f_{x}$ vanishes on a neighborhood $U_{x}$ of $x$.

Now we continue with a standard compactness argument. Finitely many of the neighborhoods $U_{x}$ cover the compact set $\operatorname{supp} \mu$, say $\operatorname{supp} \mu \subseteq U_{x_{1}} \cup$ $\cdots \cup U_{x_{n}}$. Clearly, there are strictly positive constants $r_{1}$ and $r_{2}$ such that $\mu\left(r_{1} f_{x_{1}}\right)=\mu\left(r_{2} f_{x_{2}}\right)$, which implies that $r_{1} f_{x_{1}} \wedge r_{2} f_{x_{2}} \in E$. Continuing this process inductively yields constants $r_{1}, \ldots, r_{n}>0$ such that $f_{0}=r_{1} f_{x_{1}} \wedge \cdots \wedge$ $r_{n} f_{x_{n}} \in E$. This function $f_{0}$ vanishes on $\operatorname{supp} \mu$ and is strictly positive at $x_{0}$.

2.5. Corollary. Let $K$ be a compact Hausdorff space, and let $E \subseteq C(K)$ be a linear subspace that separates the points of $K$. Assume that $1 \in \bar{E}$. If there is a strictly positive functional $\phi: E \rightarrow \Re$ such that $E$ is a $\phi$-lattice, then $E$ is a sublattice of $C(K)$.

\section{ARCHIMEDEAN ORDERED VECTOR SPACES}

In this section, we will show that every Archimedean ordered vector space that is also a $\mu$-lattice for a certain strictly positive functional $\mu$ is actually a vector lattice. 
We will start our discussion with order unit spaces. This special case can be reduced to 2.5 . The arguments are to a large extend standard in the theory of compact convex sets and boundary integrals, see also [1]. If $(E, e)$ is an Archimedean ordered vector space with an order unit $e$, let $S(E)$ denote the state space of $E$, that is $S=\{\phi: E \rightarrow \Re \mid \phi$ is positive, and $\phi(e)=1\}$. When equipped with the weak-*-topology, $S$ is a compact convex set. The following result is a consequence of Herve's Theorem (see also Proposition 1.4.1 in [1]); the proof is a variation of the proof of Theorem II.1.9 in [1].

3.1. Theorem. Let $(E, e)$ be an Archimedean ordered vector space with order unit $e$, and let $p \in \partial S(E)$ be an extreme point of $S(E)$. If $a, b \in E$ and $a \vee b$ exists, then $p(a \vee b)=\max \{p(a), p(b)\}$. Moreover, if $\phi$ belongs to the weak-*-closure of $\partial S(E)$, then $\phi(e)=1$ and $\phi$ preserves all existing suprema.

Proof. The proof of the first half of the theorem is an exact copy of the corresponding part of Theorem II.1.9 in [1]; one only has to remark that it is enough to postulate the existence of $a \vee b$ and that the full lattice structure is never used. For the second half, let $\left(p_{i}\right)_{i \in I}$ be a net of extreme points of $S(E)$ that converges to $\phi$ in the weak-*-topology. Then $\phi(e)=\lim p_{i}(e)=\lim 1=1$ and $\phi(a \vee b)=\lim p_{i}(a \vee b)=\lim \max \left\{p_{i}(a), p_{i}(b)\right\}=\max \left\{\lim p_{i}(a), \lim p_{i}(b)\right\}=$ $\max \{\phi(a), \phi(b)\}$ whenever $a \vee b$ exists.

3.2. Theorem. Let $(E, e)$ be an Archimedean ordered vector space with order unit $e$. Let $K$ be the weak-*-closure of $\partial S(E)$. Then $(E, e)$ is order isomorphic to a subspace $F$ of $C(K)$ under an isomorphism $\Psi: E \rightarrow F$ such that

(i) $\Psi(e)=1$.

(ii) If $a \vee b$ exists in $E$, then the pointwise supremum of $\Psi(u)$ and $\Psi(v)$ belongs to $F$ and $\Psi(a \vee b)=\Psi(a) \vee \Psi(b)$.

(iii) $F$ separates the points of $K$.

In addition, if $E$ admits a strictly positive functional $\mu$ such that $E$ is a $\mu$-lattice, then $F$ is a $\mu \circ \Psi^{-1}$-lattice.

Proof. The proof of this result is an easy application of Kadison's Theorem (see also II.1.8 in [1]) in connection with 3.1.

We now can show the following

3.3. Corollary. Let $(E, e)$ be an Archimedean ordered vector space with order unit $e$. If there is a strictly positive measure $\mu$ on $E$ such that $E$ is a $\mu$-lattice, then $E$ is a vector lattice.

Proof. This statement follows from 2.5 and 3.2 and the observation that an ordered vector space that is order isomorphic to a vector lattice is a vector lattice in its own right.

Before we prove our main result, we need one additional lemma.

3.4. Lemma. Let $E$ be an ordered vector space, and assume that the positive cone $C=\{x \in E: 0 \leq x\}$ contains at least one nonzero element. If there is $a$ strictly positive linear functional $\mu: E \rightarrow \Re$ such that $E$ is a $\mu$-lattice, then the positive cone is generating in the sense that $E=C-C$.

Proof. Let $0 \neq u \in C$, and let $x \in E$ be arbitrary. If $\mu(x) \geq 0$, then 0 , $x \leq x \vee(\mu(x) / \mu(u)) \cdot u \in E$. It follows that

$$
x=(\mu(x) / \mu(u)) \cdot u-((\mu(x) / \mu(u)) \cdot u-x) \in C-C .
$$


If $\mu(x) \leq 0$, then $\mu(-x) \geq 0$, hence $-x \in C-C$ by the previous argument, and thus $x \in C-C$, since $C-C$ is a linear subspace.

We are now able to show

3.5. Theorem. Let $E$ be an Archimedean ordered vector space, and assume that the positive cone of $E$ is generating. If there exists a strictly positive functional $\mu$ on $E$ such that $E$ is a $\mu$-lattice, then $E$ is a vector lattice.

Proof. For each positive $u \in E$ let $E_{u}=\bigcup_{n>0}\{x \in E: n u \leq x \leq n u\}$ be the order ideal generated by $u$. Then $\left(E_{u}, u\right)$ is an Archimedean ordered vector space with order unit $u$. Moreover, the restriction of $\mu$ to $E_{u}$ defines a strictly positive functional on $E_{u}$, and $E_{u}$ is a $\mu$-lattice with respect to this functional. Hence each $E_{u}$ is a vector lattice by 3.3. Since $E=\bigcup_{u>0} E_{u}$, it can be expected that $E$ is also a vector lattice; we just have to show that the supremum of the element $x \in E$ with 0 does not depend on the order ideal $E_{u}$ in which it is computed. Let us denote the supremum of $x$ and 0 in $E_{u}$ by $x_{u}$. Let $u$ and $v$ be two positive elements such that $x \in E_{u} \cap E_{v}$. If $u \leq v$, then $E_{u} \subseteq E_{v}$, and hence it follows that $x_{v} \leq x_{u}$ since $x_{u} \in E_{v}$ is an upper bound of $x$ and 0 . Because $E_{u}$ is an order ideal, it follows that $x_{v} \in E_{u}$, and since $x_{u}$ is the least upper bound of $x$ and 0 in $E_{u}$, we conclude that $x_{u} \leq x_{v}$, i.e., $x_{u}=x_{v}$. If $u$ and $v$ are arbitrary positive elements such that $x \in E_{u} \cap E_{v}$, then also $x \in E_{u+v}$, and we have just argued that $x_{u}=x_{u+v}=x_{v}$. Therefore the supremum of $x$ and 0 exists in $E$, and $E$ is a vector lattice.

In connection with Rosenthal's result in [7] we now obtain

3.6. Corollary. Let $K$ be a line-compact convex set such that the nonempty intersection of two translates of $K$ is a homothet of $K$. Then $K$ is a simplex.

\section{REFERENCES}

1. E. M. Alfsen, Compact convex sets and boundary integrals, Springer-Verlag, New York, Heidelberg, and Berlin, 1971.

2. H. H. Schaefer, Topological vector spaces, Springer-Verlag, New York, Heidelberg, and Berlin, 1971.

3. __ Banach lattices and positive operator, Springer-Verlag, New York, Heidelberg, and Berlin, 1974.

4. D. G. Kendall, Simplexes and vector lattices, J. London Math. Soc. (2) 37 (1962), 365-371.

5. W. A. J. Luxemburg and A. C. Zaanen, Riesz spaces. I, North-Holland, Amsterdam and Berlin, 1971.

6. H. Rosenthal, $L^{1}$-convexity, Functional Analysis Proceedings, University of Texas, Lecture Notes in Math., vol. 1332, Springer-Verlag, Berlin, Heidelberg, and New York, 1988, pp. 156-174.

7. $\ldots$, On the Choquet representation theorem, Functional Analysis Proceedings, University of Texas, Lecture Notes in Math., vol. 1332, Springer-Verlag, Berlin, Heidelberg, and New York, 1988, pp. 1-32.

8. K. Yoshida, Functional analysis, Springer-Verlag, New York, Heidelberg, and Berlin, 1965.

Department of Mathematics, University of California, Riverside, California 92521 E-mail address: gierz@ucrmath.ucr.edu

Department of Mathematics, University of South Florida, Tampa, Flordia 33620

E-mail address: boris@gauss.math.usf.edu 